\title{
The role of mitophagy in innate immune responses triggered by mitochondrial stress
}

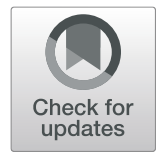

Yinjuan Song ${ }^{1}$, Yang Zhou ${ }^{2,3}$ and Xiangmei Zhou ${ }^{1 *}$ (D)

\begin{abstract}
Mitochondria are important cellular organelles involved in many different functions, from energy generation and fatty acid oxidation to cell death regulation and immune responses. Accumulating evidence indicates that mitochondrial stress acts as a key trigger of innate immune responses. Critically, the dysfunctional mitochondria can be selectively eliminated by mitophagy. The elimination of dysfunctional mitochondria may function as an effective way employed by mitophagy to keep the immune system in check. In addition, mitophagy can be utilized by pathogens for immune evasion. In this review, we summarize how mitochondrial stress triggers innate immune responses and the roles of mitophagy in innate immunity and in infection, as well as the molecular mechanisms of mitophagy.
\end{abstract}

Keywords: Mitophagy, Mitochondrial stress, Innate immunity, Infection, Mitophagy mechanisms

\section{Introduction}

The innate immune system is the first line of defense against pathogen infection. Pathogens are initially sensed by pattern-recognition receptors (PRRs) of the innate immune system, which bind to pathogen-associated molecular patterns (PAMPs), including the structural components, nucleic acids and proteins of pathogenic microorganisms [1]. The PRRs include four families: Toll-like receptors (TLRs), nucleotide oligomerization domain (NOD)-like receptors (NLRs), C-type lectin receptors (CLRs) and retinoic acid-inducible gene I (RIG-I)-like receptors (RLRs) [2, 3]. Ligand binding initiates activation of PRRs, resulting in the activation of nuclear factor-карра B (NF-кB), mitogen-activated protein kinases (MAPKs), inflammasomes and interferon regulatory factors (IRFs), and subsequent production of pro-inflammatory cytokines, chemokines, type I interferon (IFN) and co-stimulatory molecules [1]. Multiple

\footnotetext{
*Correspondence: zhouxm@cau.edu.cn

'Key Laboratory of Animal Epidemiology and Zoonosis, Ministry of Agriculture, National Animal Transmissible Spongiform Encephalopathy Laboratory, College of Veterinary Medicine, China Agricultural University, Beijing 100193, China

Full list of author information is available at the end of the article
}

studies have established crosstalk between innate immune signals and mitochondria.

Mitochondria are dynamic, double-membrane-bound eukaryotic organelles that originated from an ancient $\alpha$-proteobacterium more than $1.5-2.5$ billion years ago [4-6]. Mitochondria maintain their own genetic material, mitochondrial DNA (mtDNA), which is a small, double-stranded circular molecule that only encodes 13 necessary protein subunits of the oxidative phosphorylation system (OXPHOS), and about $99 \%$ of mitochondrial proteins are nuclear DNA encoded [7]. Mitochondria perform multiple functions in cells, which are the energy factory of the cell. In addition to energy generation, their crucial roles in metabolism include amino acid and fatty acid metabolism and biosynthesis of heme, hormones and iron-sulfur clusters. Moreover, mitochondria are involved in the regulation of apoptosis and calcium homeostasis, and reactive oxygen species (ROS) signaling [8]. Furthermore, mitochondria have been indicated to have various functions in innate immune pathways. They orchestrate signaling and effector responses to enhance immune cell activation and antimicrobial defense, and elicit inflammation in response to 
intracellular and extracellular stimuli [9]. Mitochondrial dysfunction involved in various processes closely related to abnormalities of the immune system [10]. Hence, it is important to maintain sufficient healthy mitochondria to keep the immune system in check [11].

Mitophagy is utilized by eukaryotic cells to eliminate damaged mitochondria and preserve healthy mitochondria. It is one of the major pathways of mitochondrial quality control, which monitors the function of mitochondrial and provokes a repair response when damage is discerned [12]. In 1966, mitophagy was first observed in mammalian cells by early electron microscopy studies [13]. Mitophagy is a vital selective autophagy process and subdivided into ubiquitin (Ub)-dependent or Ubindependent (receptor-dependent) pathways [14]. Accumulating evidence implicates that mitophagy keeps the immune system in check by removing stressed mitochondria [11].

Mitochondria are increasingly recognized as pivotal hubs in innate immune responses, and mitochondrial damage-associated molecular patterns (DAMPs) are key triggers in the activation of innate immune responses following a variety of stimuli that include infection, tissue damage and metabolic dysregulation [15]. Moreover, mitophagy is critical for maintaining mitochondrial function and homeostasis [16]. In this review, we summarize the emerging researches on the function of mitophagy in innate immune responses triggered by mitochondrial stress, with a specific focus on the molecular mechanisms of mitophagy and the role of mitophagy in type I IFN production, IL-1 $\beta$ responses and apoptosis.

\section{Mitochondrial stress acts as a trigger of innate immune responses \\ Mitochondrial stress}

Mitochondrial stress can be caused by various insults from environment (e.g., radiation, toxic chemicals), genetic mutations (e.g., mutations in genes for metabolic processes or repair pathways), pathogen infection (e.g., virus, bacteria) [17] and spontaneous (e.g., ROS generated as a byproduct of electron transport) [12]. These insults damage mitochondrial proteins, lipids, and DNA, resulting in loss of mitochondrial membrane integrity and membrane potential, metabolic dysfunction, alterations in energy intermediates or impairment of mitochondrial translation $[12,18,19]$. To cope with these challenges, mitochondria continuously crosstalk with the nucleus and the cytosol, activating quality-control proteases [20], lipases [21], mitochondrial unfolded protein response [22], apoptosis [23], mtDNA repair enzymes $[24,25]$, mitochondrial morphology transitions [26, 27] and mitophagy [14] to restore proper mitochondrial function.
Specifically, mitochondrial stress is characterized by impaired OXPHOS and metabolism, loss of membrane potential and increased levels of mitochondrial ROS (mtROS), etc. These processes effectively disrupt mitochondrial membrane integrity, leading to the release of DAMPs into the cytosol or extracellular environment. Mitochondrial DAMPs include mtDNA, mtROS, Nformylated peptides and cardiolipin. These mitochondrial DAMPs can activate PRRs of the innate immune system, then trigger a wide range of inflammatory responses, including inflammasome activation, production of type I IFN and pro-inflammatory cytokines and chemokines [10]. Moreover, it has been shown that mitochondrial-derived molecules (i.e., cytochrome c) are involved in apoptosis [28]. In this scenario, we will discuss how mitochondrial stress triggers inflammatory responses (i.e., IL-1 $\beta$, type I IFN responses) and how mitochondria control cell death.

\section{Mitochondrial stress and inflammasome}

Inflammasomes are an essential part of the host innate immune system. They are cytoplasmic multiprotein complex composed of the innate immune sensor molecule and the effector molecule (i.e., pro-caspase-1). Innate immune sensors including Nod-like receptor CARD domain containing 4 (NLRC4), Nod-like receptor pyrin domain containing 3 (NLRP3) or absence in melanoma 2 (AIM2) [29-32]. The requirement of adaptor molecules (i.e., apoptosis-like speck protein, or ASC) relies on the presence or absence of matching domains within the sensor and effector molecules. Some inflammasomes may require adaptor molecules that bridge the interactions of protein-protein to ultimately elicit inflammasome assembly [29, 32, 33]. The sensor, adaptor, and effector molecules of inflammasome form a large cytosolic multiprotein complex upon stimulated by its activators (e.g., pathogen infection or sterile injury), leading to the self-cleavage and activation of pro-caspase- 1 . Then, active caspase- 1 proteolytically processes prointerleukin-1 $\beta$ (pro-IL-1 $\beta$ ) and pro-IL-18, into their biologically active forms (namely IL-1 $\beta$ and IL-18), respectively $[29,30,32,33]$.

The NLRP3 inflammasome is the most extensively studied inflammasome and is activated by a wide spectrum of stimuli including microbial infection, bacterial pore-forming toxins, aluminum hydroxide, silica crystals, monosodium urate, extracellular ATP and asbestos [34-38]. It has been proposed that the activation of NLRP3 is controlled by mitochondria [39]. In 2011, Nakahira et al. found that cytosolic mtDNA contributes to the secretion of IL- $1 \beta$ and IL-18 in response to lipopolysaccharide (LPS) and ATP, and the release of mtDNA into the cytosol depends on the mtROS production [40]. mtDNA is required for NLRP3 inflammasome 
activation in murine macrophages for depletion of mtDNA (low-dose chronic treatment of ethidium bromide) attenuates IL-1 $\beta$ release and caspase- 1 activation [40]. This study first linked mtDNA to the activation of NLRP3 inflammasome. In 2012, NLRP3 was shown to bind to cytosolic oxidatively damaged or oxidized mtDNA (Ox-mtDNA) released during apoptosis causing its activation [41]. This study extended our understanding of the interplay between mtDNA and the NLRP3 inflammasome. The above results were further verified by another approach by which the genetic ablation of mitochondrial transcription factor A (TFAM) in mouse myeloid cells causes more than 95\% mtDNA deletion and prevents NLRP3 inflammasome activation [42]. Transfection of Ox-mtDNA in TFAM-ablated macrophages rescued the activity of defective NLRP3 inflammasome, suggesting that Ox-mtDNA is sufficient and necessary for NLRP3 inflammasome activation [42]. In addition to NLRP3, mtDNA may influence other inflammasomes activation: mtDNA bound specifically to NLRC4 immunoprecipitate and transfection of mitochondrial DNA directly activated the NLRC4 inflammasome [43]. Moreover, the mtDNA from patients with type 2 diabetes triggers AIM2 inflammasome-dependent caspase- 1 activation and IL-1 $\beta$ and IL-18 secretion in macrophages [44].

In 2010, the generation of ROS proved to be one of the essential elements for NLRP3 inflammasome activation [45]. In 2011, Zhou et al. found that mtROS was essential for NLRP3 inflammasome activation [39]. Although mtROS is essential, it is not sufficient to activate NLRP3. The addition of $\mathrm{H}_{2} \mathrm{O}_{2}$ (the dominant form of mtROS) cannot rescue defective NLRP3 inflammasome activity in TFAM-deleted macrophages [42]. This is in line with the fact that not all mtROS inducers (e.g., TNF) activate NLRP3 inflammasome [29, 30]. Additionally, NLRP3 inflammasome activation is partially triggered by ATP produced by mitochondria released from damaged cells [46]. The depletion of cardiolipin by knockdown of cardiolipin synthase attenuates NLRP3 inflammasome activation by reverse transcriptase inhibitor abacavir, suggesting mitochondrial cardiolipin also triggers NLRP3 inflammasome activation [47, 48]. Furthermore, some mitochondrial proteins also play a crucial role in inflammasome activation. In 2011, the research showed that voltage-dependent anion channel (VDAC) is important for NLRP3 inflammasome activation. Both ROS generation and inflammasome activation are suppressed when mitochondrial activity is dysregulated by inhibition of the VDAC [39]. The VDAC is a component of the mitochondrial permeability transition pore (MPTP) through which large molecules (e.g., mtDNA) are released into the cytoplasm [49, 50]. In 2013, the study suggested that mitochondrial antiviral signaling proteins (MAVS) is required for optimal NLRP3 inflammasome activity. MAVS mediates the recruitment of NLRP3 to mitochondria, promoting the production of IL-1 $\beta$ and the pathophysiologic activity of the NLRP3 inflammasome in vivo [51].

In summary, mitochondria play a key role in the activation of NLRP3 inflammasome. When cells are stimulated by various stress signals, mitochondria release mitochondrial DAMPs (such as mtROS, mtDNA, ATP) into the cytoplasm, which trigger the assembly of cytosolic NLRP3, ASC and pro-caspase-1 [36]. The entry of mitochondrial DAMPs into the cytoplasm may depend on VDAC $[49,50]$. The assembled NLRP3 inflammasome is recruited to the outer mitochondrial membrane and interacts with MAVS to promote inflammasome activation and IL-1 $\beta$ production [51]. Although how NLRP3 inflammasome activation is triggered is extensively investigated, the exact mechanism of its assembly and activation still needs further exploration.

\section{Mitochondrial stress and type I IFN responses}

In addition to inducing pro-inflammatory cytokines production, mtDNA accesses into the cytosol or extracellular space during mitochondrial dysfunction, and triggers expression of type I IFN and interferon-stimulated genes (ISGs) in mice and humans [52]. Although some of the mechanistic details are still unclear, the identification of mtDNA as a DAMP that induces type I IFN production is crucial for understanding the pathology of infectious and non-infectious diseases involving mitochondrial dysfunction [53]. In this section, we will discuss the roles of mtDNA in type I IFN responses.

Cytosolic mtDNA engages cyclic guanosine monophosphate-adenosine monophosphate synthase (cGAS) activation and subsequently elicits type I IFN production. cGAS is a cytosolic nucleotide synthase that binds DNA, and catalyzes ATP and GTP to synthesize cyclic guanosine monophosphate-adenosine monophosphate (cGAMP). Then, cGAMP acts as a second messenger to bind and activate the stimulator of interferon genes (STING) [54, 55]. STING further activates TANKbinding kinase 1 (TBK1) via its $\mathrm{C}$ terminal PLPLRT/SD motif [56]. TBK1 promotes IRF3 dimerization and translocation into the nucleus, where it triggers the expression of type I IFN and various ISGs [57, 58]. Specifically, it has been demonstrated that cGAS functions as the main sensor of viral and bacterial DNA in the cytoplasm of infected cells [59-61], such as Mycobacterium tuberculosis (Mtb) infected bone marrow-derived macrophages (BMDM) cGAS senses Mtb DNA in the cytosol and triggers the expression of type I IFN. Recent evidence indicates that mtDNA may also serve as an endogenous cGAS ligand in certain conditions. For instance, mitochondrial stress provoked by Mtb 
infection results in mtDNA leaking into the cytoplasm and cytosolic mtDNA contributes to IFN- $\beta$ production by activating the cGAS-STING pathway [17]. Moreover, West and colleagues found that mouse embryonic fibroblasts (MEFs) from the TFAM heterozygous knockdown $\left(\mathrm{TFAM}^{+/-}\right)$mice, exhibit disruption of mtDNA stability, which leads to mtDNA release into the cytosol, where it engages the DNA sensor cGAS and promotes STINGIRF3-dependent signaling to elevate ISGs expression and potentiate type I IFN responses [62].

Furthermore, previous studies demonstrated that mtDNA release during $\mathrm{Bcl}-2$ associated-X protein (Bax) and BCL-2 homologous antagonist/killer (Bak)-mediated apoptosis elicits cGAS-STING signaling and induces type I IFN production. Inhibition of apoptotic caspase-9/ $-3 /-7$ cascade enhances the type I IFN responses triggered by cytosolic mtDNA, suggesting that apoptotic caspase-9/-3/-7 cascade suppress mtDNA-mediated type I IFN production during the apoptosis process [63, 64]. Recently, apoptotic caspase-3 was shown to inhibit type I IFN production via cleaving and inactivating cGAS, MAVS and IRF3 [65]. Notably, active caspase-1 was also shown to cleave cGAS in macrophages and inhibit type I IFN expression [66]. These results collectively indicate that the activation of cGAS triggered by host-derived mtDNA is likely to be a strictly regulated process to hinder uncontrolled type I IFN production that might otherwise cause pathological disease (i.e., autoimmunity disease).

Toll-like receptor 9 (TLR9) was the first identified nucleic acid-sensing TLR and recognizes hypo-methylated cytosine-guanosine (CpG) motifs in the DNA. It is expressed primarily in monocytes, macrophages, plasmacytoid dendritic cells (pDCs) and B lymphocytes [67]. In addition to recognizing bacterial or viral DNA, mounting literature supports the notion that mtDNA is a potent ligand for TLR9, for bacterial DNA and mtDNA share hypo-methylated $\mathrm{CpG}$ motifs that can be recognized by TLR. For example, the plasma from mice and patients with nonalcoholic steatohepatitis (NASH) contains high levels of mtDNA that has the ability to activate TLR9 [68]. And, mtDNA contributes to the initiation of sterile systemic inflammatory response syndrome (SIRS) via activating the TLR9/NF-kB pathway and inducing proinflammatory cytokines expression [69]. In addition to eliciting pro-inflammatory responses, extracellular oxidized mtDNA has been shown to induce the TLR9- and IRF7-dependent production of IFN- $\alpha$ to augment type I IFN responses in pDCs [70].

From the mentioned above, mtDNA appears to be a potent ligand of the cGAS and TLR9. mtDNA influences type I IFN responses and pro-inflammatory responses in multiple pathological states, including infectious diseases, autoimmunity, and other illnesses in which mitochondrial integrity is damaged. Even though previous studies proposed mitochondria-derived vesicles (MDVs) [52], MPTP opening [40, 71] and Bax/Bak proteins oligomerization [72] have been associated with the release of mtDNA into the cytoplasm, the precise mechanisms underlying mtDNA escape from damaged mitochondria remain unclear.

\section{Mitochondria control cell death}

Mitochondria play a crucial role in the regulation of apoptotic cell death. These organelles not only modulates intrinsic apoptosis triggered by alterations of intracellular homeostasis but also participates in the regulation of the extrinsic apoptotic response to external stimuli [73]. Besides apoptosis, mitochondria also control non-apoptotic types of programmed cell death, including regulated necrosis [74]. The mechanisms of mitochondria control cell death are extensively reviewed elsewhere [4, 28]. The main factors carrying out apoptosis include Bax and Bak, which trigger the outer mitochondrial membrane (OMM) permeabilization upon activation, leading to the irreversible release of intermembrane space proteins (e.g., cytochrome c), subsequent activation of caspase-9/-3/-7 and apoptosis [75]. Apoptosis is an important innate cellular defense mechanism that prevents the growth of intracellular microbes, including virulent Mtb [76]. The crosstalk between mitochondrial apoptosis pathway and various pathogens infection was reviewed in 2020 by Varnesh Tiku et al. [77]. Here, we focus on the mechanisms of mycobacteriuminduced apoptosis and necrosis, and the role of mitochondria in it.

Pathogenic intracellular bacteria have evolved multiple strategies to target host cells to either inhibit apoptosis to maintain their survival and growth or promote apoptosis. The previous study suggested that Mtb differentially regulates myeloid cell leukemia-1(Mcl-1) and Bax expression through peroxisome proliferator-activated receptor (PPAR) gamma to limit apoptosis [76]. Mtb heparin-binding hemagglutinin (HBHA) targets to mitochondria and then caused mitochondrial damage and oxidative stress, which eventually lead to Mtb infected or HBHA-treated macrophages apoptosis [78]. In addition to Mtb, Mycobacterium avium MAV2054 protein induces caspase-dependent macrophage apoptosis by targeting mitochondria. The apoptotic response and ROS production were significantly increased and mitochondrial transmembrane potential $(\Delta \Psi \mathrm{m})$ was decreased in macrophages infected with Mycobacterium smegmatis expressing MAV2054 [79]. A recent study reported that $M$. bovis infection upregulates the expression of the Kallikrein 12 (KLK12), and knockdown of KLK12 results in a significant downregulation of autophagy and apoptosis in M. bovis-infected BMDMs. Furthermore, 
KLK12-mediated regulation of apoptosis involves BAX/ Bcl-2/cytochrome c/caspase 3 pathways [80]. Mycobacterium fortuitum infection induces $\mathrm{Ca}^{2+}$ mobilization from the endoplasmic reticulum (ER) to mitochondria, leading to increased mitochondrial $\mathrm{Ca}^{2+}$ load, MPTP opening, altered mitochondrial membrane potential $(\Delta \Psi \mathrm{m})$, cytochrome $\mathrm{c}$ release, and eventually activation of the caspase-9/-3 mediated apoptosis in fish macrophage [81].

Mycobacterial infection leads to apoptosis or necrosis depends on differences in mitochondrial membrane perturbation induced by attenuated or virulent strains. The virulent $\mathrm{H} 37 \mathrm{Rv}$ promotes necrosis via inducing substantial alterations to mitochondrial transmembrane potential $(\Delta \Psi \mathrm{m})$, while the attenuated H37Ra infection predominantly leads to apoptosis [82]. A previous study reported that Mtb Rv2626c gene is associated with the host cell necrosis. When Rv2626c gene is deleted from the genome of Mtb, THP-1 cells undergo less necrosis following infection with Rv2626c gene-deleted Mtb, and overexpression of Rv2626c promotes the host cell necrosis at early time points [83]. However, the mechanism by which mycobacterium promotes necrosis has not been fully elucidated. In summary, mitochondria have an important role in intrinsic apoptosis as well as necrosis.

As described above, mitochondria play key roles in the innate immune responses and mitochondrial stress causes inflammasome activation, type I IFN production and induction of apoptosis (Fig. 1). Meanwhile, mounting studies demonstrated that mitophagy can prevent inflammasome activation by clearing damaged mitochondria [84-87]. Next, we will summarize the studies on the molecular mechanisms of mitophagy, and the function of mitophagy in innate immune responses triggered by mitochondrial stress.

\section{Molecular pathways of mitophagy}

Autophagy is a process that delivery of cytoplasmic cargo to the lysosome for degradation and highly conserved in eukaryotes. There are at least three different autophagy forms (i.e., chaperone-mediated autophagy, microautophagy, and macroautophagy) which differ in the way of cargo delivered to the lysosome. Macroautophagy (herein referred to as autophagy) is the crucial degradative mechanism adopted by eukaryotic cells to sustain nutrient homeostasis and organellar quality control [88-90]. Autophagy was previously described as a non-selective process in response to starvation. However, it is now clear that autophagy can selectively degrade damaged or dysfunctional organelles, remove aggregated proteins and eliminate intracellular pathogens [91]. Several types of selective autophagy have been described, such as aggrephagy (removal of aggregated proteins), mitophagy (turnover of damaged mitochondria), pexophagy (degradation of peroxisomes), ribophagy (turnover of damaged ribosomes), reticulophagy (degradation of the endoplasmic reticulum) and xenophagy (elimination of pathogens) [90, 91].

The process of autophagy that selectively degrades mitochondria is called mitophagy, which is critical for mitochondrial quality control and the maintenance of normal cellular physiology. Cells possess multiple mitophagy mechanisms, and different stimuli promote mitophagy through multiple signal cascades in different cellular environments [92]. In general, mitophagy regulatory pathways are classified as ubiquitin (Ub)-dependent or Ub-independent (receptor-dependent) [14].

\section{Pink1-Parkin-mediated mitophagy}

The phosphatase and tensin homologue (PTEN)-induced putative kinase 1 (Pink1)- parkin RBR E3 ubiquitin protein ligase (Parkin) mediated mitophagy is the most extensively studied. Ub-dependent pathway is employed to clear damaged mitochondria and is generally related to the alteration of mitochondrial transmembrane potential [93]. Pink1 is a mitochondrial Ser/Thr kinase, encoded in nuclear DNA, exists as a precursor form in the cytosol, and then transported into mitochondria [94]. Under physiological conditions, Pink1 is transported into the inner mitochondrial membrane (IMM), and cleaved by several IMM-resident proteases include presenilinassociated rhomboid-like (PARL). Cleaved Pink1 is retro-translocated into the cytoplasm and subsequently degraded by the proteasome through the $\mathrm{N}$-end rule pathway [94]. Following mitochondrial membrane potential dissipation, the translocation of cytosolic Pink1 into the mitochondrial matrix is prevented, leading to the stabilization of Pink1 on the outer membrane of the damaged mitochondria [94]. Concomitantly, Pink1 recruits Parkin to the mitochondrial surface and triggers its E3 ligase activity through a circuit of modifications including phosphorylation of both Parkin and ubiquitin [95]. Subsequently, Parkin mediates poly-ubiquitination of several OMM proteins including VDAC1 and mitofu$\sin 1 / 2(M F N 1 / 2)$. Polyubiquitinated proteins are identified by several adaptor molecules, including SQSTM1/ p62, optineurin (OPTN), and nuclear domain 10 protein 52 (NDP52, also known as CALCOCO2), facilitating their recognition by light chain 3 (LC3) and mitophagosome (mitochondria-specific autophagosome) formation [96]. Importantly, phosphorylated ubiquitin (pSer65-Ub and pSer57-Ub), functions as potent Parkin activator, leading to enhanced ubiquitination of proteins at the OMM and initiation of mitophagy [97-99]. Therefore, Pink1-mediated phosphorylation and Parkin-mediated ubiquitination form a feedforward mechanism of mitophagy [100, 101]. 


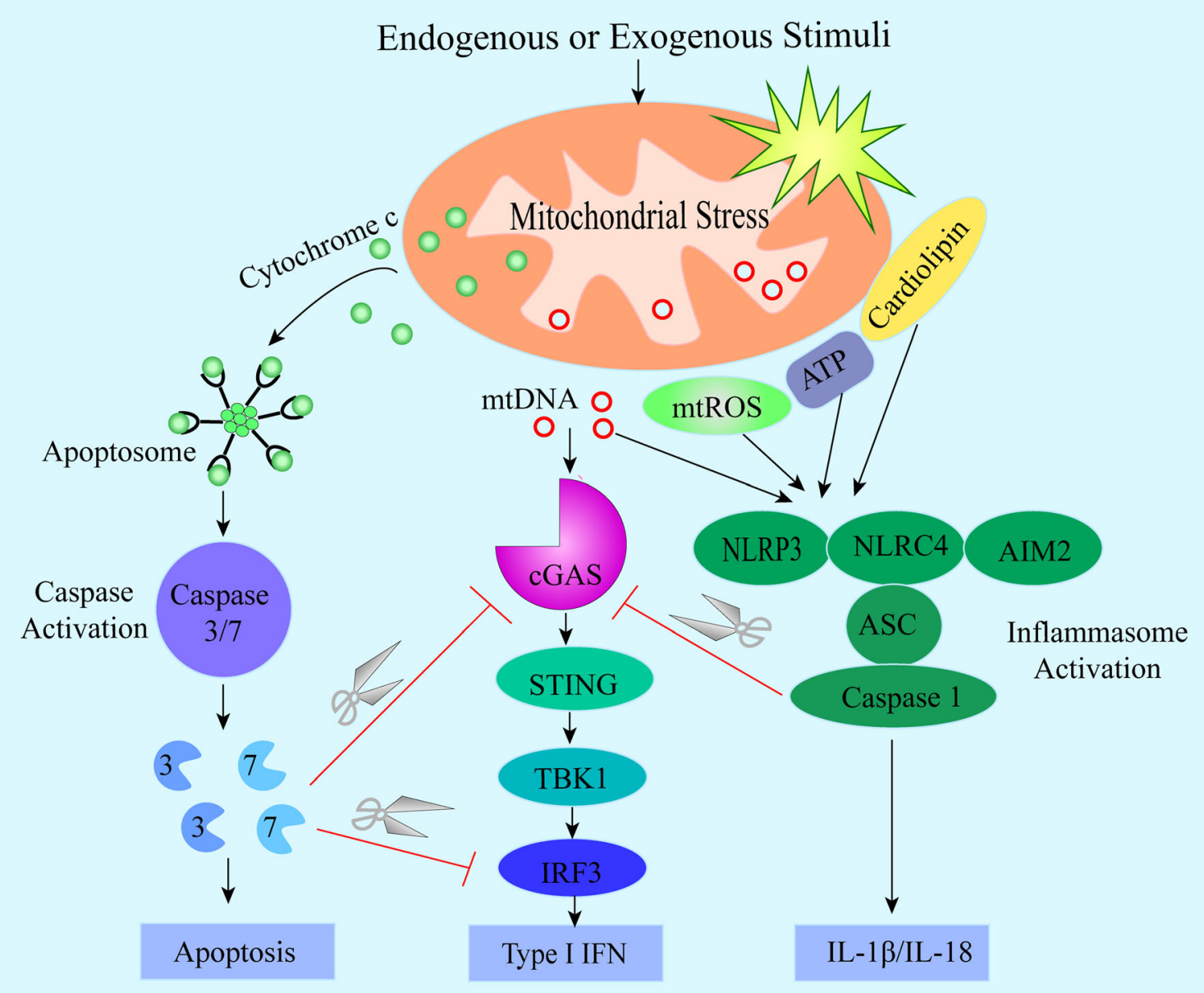

Fig. 1 Mitochondrial stress acts as a trigger of innate immune responses. Cell and tissue stress initiated by endogenous or exogenous stimuli can directly or indirectly cause mitochondrial stress. Mitochondrial stress leads to mtROS, mtDNA and ATP release into the cytosol or extracellular milieu, cardiolipin externalization and cytochrome $\mathrm{c}$ release into the cytosol. Cytosolic mtDNA activates the cGAS-STING pathway, resulting in the production of type I IFN. Moreover, mtROS, mtDNA, cardiolipin and ATP engage in the activation of inflammasome leading to IL-1 3 and IL-18 processing and secretion. Cytosolic cytochrome $\mathrm{c}$ triggers activation of apoptotic caspase-3/- 7 , and which inhibits type I IFN production via cleaving CGAS and IRF3. Similarly, caspase-1 also can cleave cGAS to prevent type I IFN expression

Notably, the negative regulatory mechanisms against Parkin-mediated ubiquitination have been reported, with the discovery of several deubiquitinases including USP8, USP15, USP30 and USP35 that are able to cause the deubiquitination of Parkin and/or OMM proteins [102107]. Furthermore, PTEN-Long (PTEN-L) is a novel negative regulator of mitophagy through its protein phosphatase activity inhibiting phosphorylated ubiquitin (pSer65-Ub). Meanwhile, it can effectively prevent Parkin mitochondrial translocation, reduce Parkin phosphorylation, maintain its inactive conformation, and inhibit its E3 ligase activity [108]. Therefore, the delicate balance between ubiquitination and deubiquitination events regulates cellular homeostasis, suggesting polyUb chains may serve as "eat me" signals to damaged mitochondria.
Pink1-Parkin-mediated mitophagy pathway interplays with other mitochondrial quality control mechanisms, such as mitochondrial dynamics, to maintain cellular homeostasis. Mitochondrial dynamics refers to the dynamic changes of mitochondria undergoing fission and fusion to maintain their shape, distribution and size. Mitochondrial fusion is assured by Mfn1 and Mfn2 and optic atrophy 1 (OPA1), which mediate OMM and IMM fusion, respectively. Mitochondrial fission is regulated by the cytosolic GTPase-protein dynamin-related protein 1 (DRP1) [109]. Pink1 serves as a pro-fission signal, which indirectly activates DRP1 in response to damage, and promotes fission of dysfunctional mitochondria, thereby segregating damaged mitochondria for mitophagy [110]. The ubiquitination of Mfn1 and Mfn2 are induced by Parkin during membrane depolarization and cause their 
degradation in a proteasome- and p97 - dependent manner [111]. In turn, Mfn2 mediates Parkin recruitment to damaged mitochondria in a Pink1-dependent manner. Depletion of Mfn2 in mouse cardiac myocytes inhibits depolarization-elicited translocation of Parkin to mitochondria and prevents mitophagy [112]. Recent studies suggest that Mfn2 is a mitochondria-ER tether that acts as a suppressor of mitophagy through the ability to link OMM to ER, which may limit the approachability of other ubiquitinated substrates to Pink1 and Parkin [113, 114].

In addition to Parkin, other E3 ubiquitin ligases are also implicated in mitophagy regulation, including HUWE1 [115], autocrine motility factor receptor (AMFR/ Gp78) [116], SMAD-specific E3 ubiquitinprotein ligase 1 (SMURF1) [117], mitochondrial E3 ubiquitin-protein ligase 1 (MUL1) [118], seven in absentia homolog 1 (SIAH1) [119], and Ariadne RBR E3 Ub protein ligase 1 (ARIH1), also known as HHARI [120] and MARCH 5 [121]. Once recruited on the OMM, they generate ubiquitin chains, triggering recruitment of several autophagy adaptor proteins, including OPTN, NDP52 and p62. Autophagy adaptor proteins interact directly with LC3 through their LC3-interacting region (LIR) motifs, initiating mitophagosome formation.

\section{Ub-independent mitophagy}

In addition to Ub-dependent mitophagy, several LC3interacting region (LIR) containing mitophagy receptors can directly induce Ub-independent mitophagy, including BCL2 interacting protein 3 (BNIP3) [122], BCL2 interacting protein 3 like (BNIP3L/NIX) [123], FUN14 domain-containing protein 1 (FUNDC1) [124], BCL2 like 13 (BCL2L13) [125], and FK506 binding protein 8 (FKBP8) [126] in mammals. These mitophagy receptor proteins are located on the OMM and interact directly with LC3 via their LIR motifs, mediating mitochondrial clearance. Of note, the recent research identified that NLRX1 is a novel mitophagy receptor for Listeria monocytogenes (L. monocytogenes)-induced mitophagy [127]. NLRX1 is the only mitochondrially localized member of the NLR family $[128,129]$, which contains an LIR motif for LC3 binding that was essential for L. monocytogenesinduced mitophagy.

BNIP3, NIX and BCL2L13 belong to the BCL2 family with pro-apoptotic activity. In addition to its apoptotic functions, BNIP3, NIX and BCL2L13 are also implicated in the initiation of autophagy. In erythroid cells, NIX is required for a specialized type of autophagy that targets mitochondria for elimination. Similarly, BNIP3 regulates mitophagy in response to hypoxia [130]. Bcl2L13 has been shown to induce mitochondrial fission and mitophagy in mammalian cells [125]. BNIP3- and NIXmediated mitophagy promotes the generation of natural killer cell memory [131]. Homodimerization of BNIP3 is a requirement for its interaction with $\mathrm{LC} 3$ and this interaction is regulated by the phosphorylation on Ser17 and Ser24 near the LIR motif [132, 133]. NIX-mediated mitophagy leads to a shift in metabolism towards the glycolysis necessary for retinal ganglion cell (RGC) neurogenesis. Moreover, NIX-mediated mitophagy also regulates the polarization of M1 macrophages, which undergo glycolysis-dependent differentiation during the inflammatory response [134]. NIX-mediated mitophagy protects against ischemic brain injury independent of PARK2, and phosphorylation of NIX at serine 81 is critical for NIX-mediated mitophagy [135]. Furthermore, NIX-mediated mitophagy plays a critical role in $\mathrm{CD}^{+} \mathrm{T}$ cell effector memory formation by regulating mitochondrial superoxide dependent hypoxia-inducible factor 1alpha (HIF1a) protein accumulation and fatty acid metabolism [136]. Recent research reported that NIX and FUNDC1-mediated mitophagy play a critical role in the formation of a functional mature mitochondrial network upon the differentiation of progenitor cells [137].

FUNDC1 is a conserved mitophagy receptor that promotes damaged or superfluous mitochondrial clearance during hypoxia in mammalian cells [124]. FUNDC1 promotes unc-51 like autophagy-activating kinase 1 (ULK1) recruitment to damaged mitochondria. In turn, ULK1 interacts with FUNDC1, phosphorylating it at serine 17, which enhances FUNDC1 binding to LC3 [138]. In addition, FUNDC1 interacts with both DRP1 and OPA1 to coordinate mitochondrial fission or fusion and mitophagy [139]. Further, it has been demonstrated that FUNDC1 is a novel MAM protein required for hypoxiainduced mitochondrial fission and mitophagy [140]. As mentioned above, NIX, BNIP3, and FUNDC1 regulate mitophagy in response to hypoxia. Although the crosstalk between FUNDC1, BNIP3, and NIX has not been fully clarified, their coordinating effects ensure the efficiency of mitochondrial quality control and cellular homeostasis. In addition, FKBP8 is also required for mitophagy under hypoxic stress [141].

Of note, certain IMM proteins have been shown to act as mitophagy receptors under stress conditions, such as cardiolipin and prohibitin2 (PHB2), which interact with lipidated LC3 via LIR motifs to promote the engulfment of defective mitochondria [142, 143]. PHB2 is an IMM protein that plays a role in development, lifespan regulation, and diverse cellular processes, including mitochondrial dynamics [144]. The interaction between PHB2 and LC3 requires OMM rupture and PHB2 cytoplasmic exposure [145]. Cardiolipin is an inner mitochondrial membrane phospholipid. Mitochondrial damage caused the externalization of cardiolipin to the mitochondrial surface. The redistribution of cardiolipin serves as an "eat-me" signal for the elimination of damaged mitochondria from neuronal cells [142]. 
It is worth mentioning that there is a crosstalk between Pink1-Parkin-mediated mitophagy and Ubindependent mitophagy [146]. It has been identified that PHB2 is required for Parkin-induced mitophagy in mammalian cells and for the clearance of paternal mitochondria after embryonic fertilization in Caenorhabditis elegans (C. elegans) [143]. The latest research reveals that PHB2-mediated mitophagy depends on the mitochondrial inner membrane protease PARL, which interacts with PHB2 and is activated after PHB2 depletion. Upon mitochondrial membrane depolarization or misfolded protein aggregation, PHB2 depletion causes the cleavage of Pink 1 by activating PRAL, which prevents the mitochondrial recruitment of Parkin, ubiquitin and OPTN, leading to an inhibition of Pink1-Parkindependent mitophagy [147].

In conclusion, the diversity of mitophagy receptors and adaptor molecules reflects the existence of a compensatory mechanism that can regulate mitochondrial homeostasis in response to a variety of intracellular and extracellular stimulus signals (Fig. 2). The complex interactions between mitophagy mechanisms ensure energy metabolism and cellular homeostasis. Therefore, maintaining mitochondrial function via fine-tuning mitophagy and mitochondrial dynamics is essential for cellular and organismal survival.

\section{The role of mitophagy in innate immunity and infection \\ Mitophagy and inflammasome}

As mentioned above, it has been demonstrated that damaged mitochondria play a crucial role in the activation of NLRP3 inflammasome by releasing mitochondrial DAMPs, such as mtROS and mtDNA. Therefore, mitophagy may be a key regulator of NLRP3 inflammasome activation, as it is a process that eliminates damaged or aberrant mitochondria [148]. This notion was experimentally demonstrated in a study showing that the deletion of autophagy protein Beclin 1 and LC3B in BMDM results in the accumulation of defective mitochondria, the increase of mtROS and an increase in mtDNA cytosolic release, which subsequently enhances NLRP3 inflammasome activation [40]. Consistent with these in vitro results, autophagy-deficient mice $\left(\right.$ Mapllc $3 b^{-1-}$ mice) were more sensitive to bacterial sepsis-induced death, which is due to higher IL- $1 \beta$ secretion than wildtype mice [40]. These findings are consistent with an earlier report suggesting that the loss of the autophagy protein autophagy-related 16-like 1 (Atg16L1) elevates endotoxin-induced IL-1 $\beta$ production [149]. The above studies indicate that autophagy inhibits inflammasome activation and subsequent IL-1 $\beta$ secretion. In recent years, studies have further proved that mitophagy can inhibit inflammasome activation. NF- $\mathrm{KB}$ restricts NLRP3 inflammasome activation through p62-dependent mitophagy. P62-depleted macrophages display significant mitochondrial damage and excessive IL- $1 \beta$-dependent inflammation [87]. Moreover, macrophage stressinducing protein SESN2 inhibits the NLRP3 inflammasome activation through inducing mitophagy [85]. Recently researches showed that OPTN inhibits the activation of NLRP3 inflammasome by enhancing mitophagy [150], and FUNDC1-mediated mitophagy suppresses hepatocarcinogenesis via inhibition of inflammasome activation [151]. Above studies show that mitophagy inhibits NLRP3 inflammasome activation by eliminating damaged mitochondria. Recent study reported that rabbit haemorrhagic disease virus (RHDV) infection induces Pink1-Parkin-mediated mitophagy and NLRP3 inflammasome activation [152]. Moreover, in 2019, $\mathrm{Li}$ and colleagues demonstrated that Parkin regulates activation of NLRP3 inflammasome, they found that Parkin deficiency enhances mtROS-mediated NLRP3 inflammasome activation and promotes viral clearance [153]. However, it has been shown that active caspase-1 can inhibit mitophagy by cleaving Parkin [154], suggesting that there may exist a mutual regulation mechanism between inflammasome and mitophagy.

\section{Mitophagy and type I IFN}

Type I IFN, including IFN- $\alpha$ and IFN- $\beta$, can activate intracellular antimicrobial programs and influence the development of innate and adaptive immune responses [155]. In recent years, some studies have reported that some viruses can directly or indirectly trigger mitophagy, and control mitophagy process through different strategies, thereby weakening the innate immune response by inhibiting the production of type I IFN or apoptosis, and enabling the virus to promote continuous infection [156]. Such as Measles virus of the Edmonston strain (MV-Edm) triggers p62-mediated mitophagy to suppress the production of type I IFN and enhances viral replication [157]. Parkin is able to recruit the linear ubiquitin assembly complex to mitochondria and abrogates IFN- $\beta$ production during $\mathrm{HBV}$ infection, which indicates that HBV usurps Parkin to impair the cellular antiviral response [158]. Moreover, matrix protein $M$ of human parainfluenza virus type 3 (HPIV3) directly interacts with $\mathrm{Tu}$ translation elongation factor mitochondrial (TUFM) and autophagy protein LC3 to induce Pink1Parkin-independent mitophagy, thereby inhibiting host type I IFN responses [159]. Furthermore, it was demonstrated that glycoprotein (Gn) of the Hantavirus (HTNV) translocates to mitochondria and interacts with TUFM, recruiting LC3B and promoting mitophagy, subsequently inhibits type I IFN responses [160]. The above research indicates that mitophagy contributes to the regulation of type I IFN through clearing dysfunctional mitochondria. 


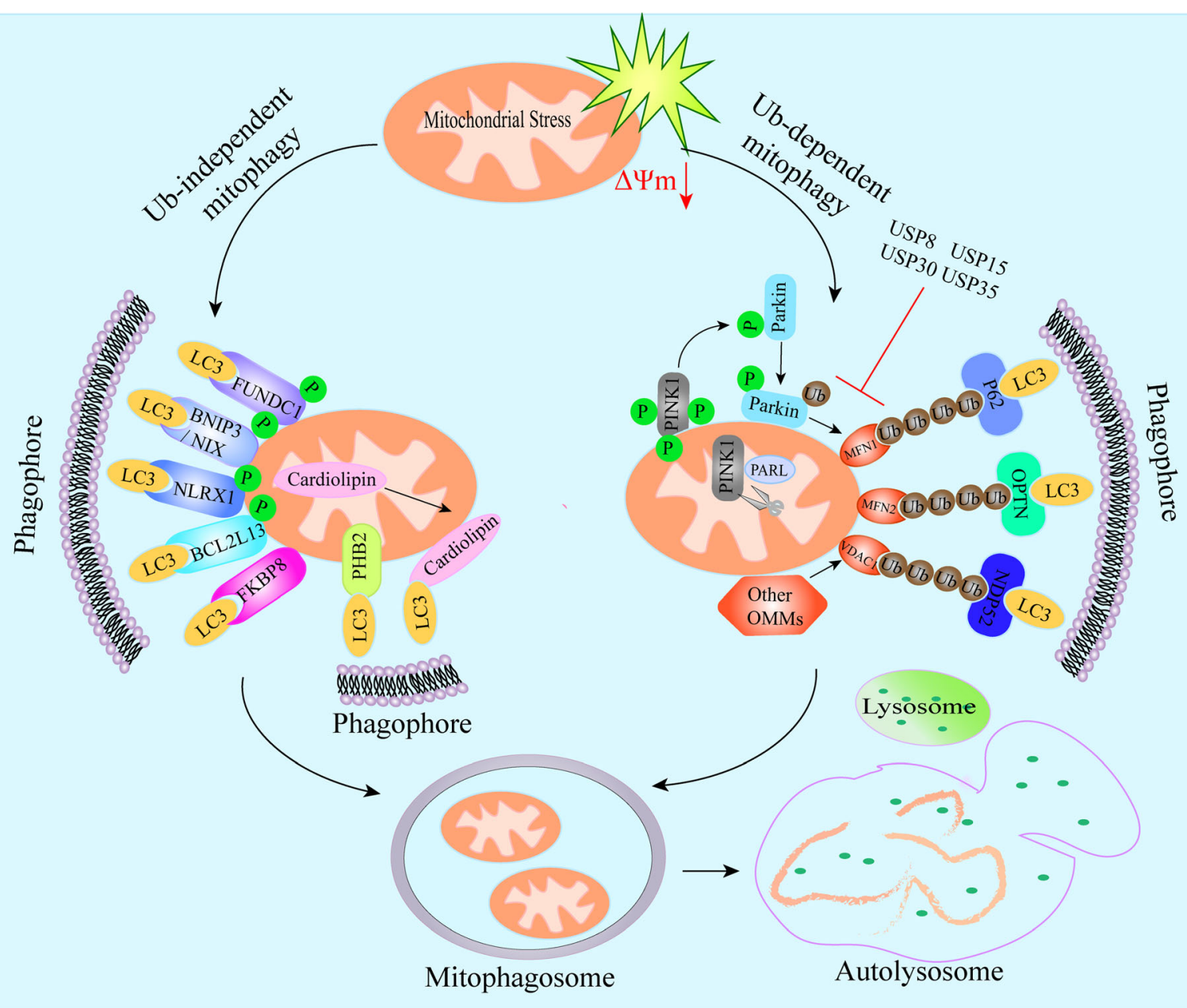

Fig. 2 Molecular mechanisms of mitophagy. Ub-dependent mitophagy usually refers to Pink1-Parkin-mediated mitophagy. Upon mitochondrial depolarization, full-length Pink1 accumulates on the OMM and recruits Parkin to the mitochondrial surface. Pink1 triggers Parkin E3 ligase activity through a circuit of modifications including phosphorylation of both Parkin and ubiquitin. Then, parkin ubiquitinates various OMM proteins including VDAC1 and MFN1/2. Polyubiquitinated proteins can be recognized by several adaptor molecules, including p62, OPTN and NDP52. These proteins interact with lipidated LC3 via the LIR motif to promote the encapsulation of damaged mitochondria by the autophagosome. In addition, deubiquitinase such as USP8, USP15, USP30, USP35 can cause the deubiquitination of Parkin and OMM proteins. Ub-independent mitophagy depends on several LIR containing mitophagy receptors, including FUNDC1, BNIP3, NIX, BCL2L13, FKBP8 and NLRX1. Besides, IMM proteins (such as cardiolipin and PHB2) interact with lipidated LC3 through the LIR motif, thereby promoting the phagocytosis of defective mitochondria. Overall, mitophagy adaptors and receptors bind to LC3 bound on the phagophores for further expansion and closure to form mitophagosomes, subsequently, mitophagosomes fuse with lysosomes form autolysosome for mitochondrial degradation

In addition, a previous study has unraveled that in the absence of N-terminal truncated isoforms of MAVS, blocking Nix-mediated mitophagy causes the stabilization of full-length MAVS and induces the subsequent secretion of type I IFN and other proinflammatory cytokines [161]. In 2018, a study reported that Parkin and Pink1 mitigate STING-induced inflammation, while STING is a central regulator of type I IFN responses to cytosolic DNA. The study supports a role for Pink1-and parkin-mediated mitophagy in suppressing innate immunity [162]. Hence, mitophagy can regulate type I IFN production by eliminating damaged mitochondria.

\section{Mitophagy and apoptosis}

Mitochondrial dynamics is integrally linked to apoptosis [23]. In 2013, Kim and colleagues reported that hepatitis $B$ virus (HBV) attenuates mitochondrial apoptosis by shifting the balance of mitochondrial dynamics toward fission and mitophagy. They found that silence of Parkin expression induces extensive cytochrome $\mathrm{c}$ release from mitochondria to cytoplasm and promotes cleavage of caspase-3 and poly (ADP-Ribose) polymerase (PARP), activation of caspase-3/-7, and promotes intrinsic apoptosis [163]. Similarly, in 2014, it has been shown that HCV promotes Pink1-Parkin-mediated mitophagy, and 


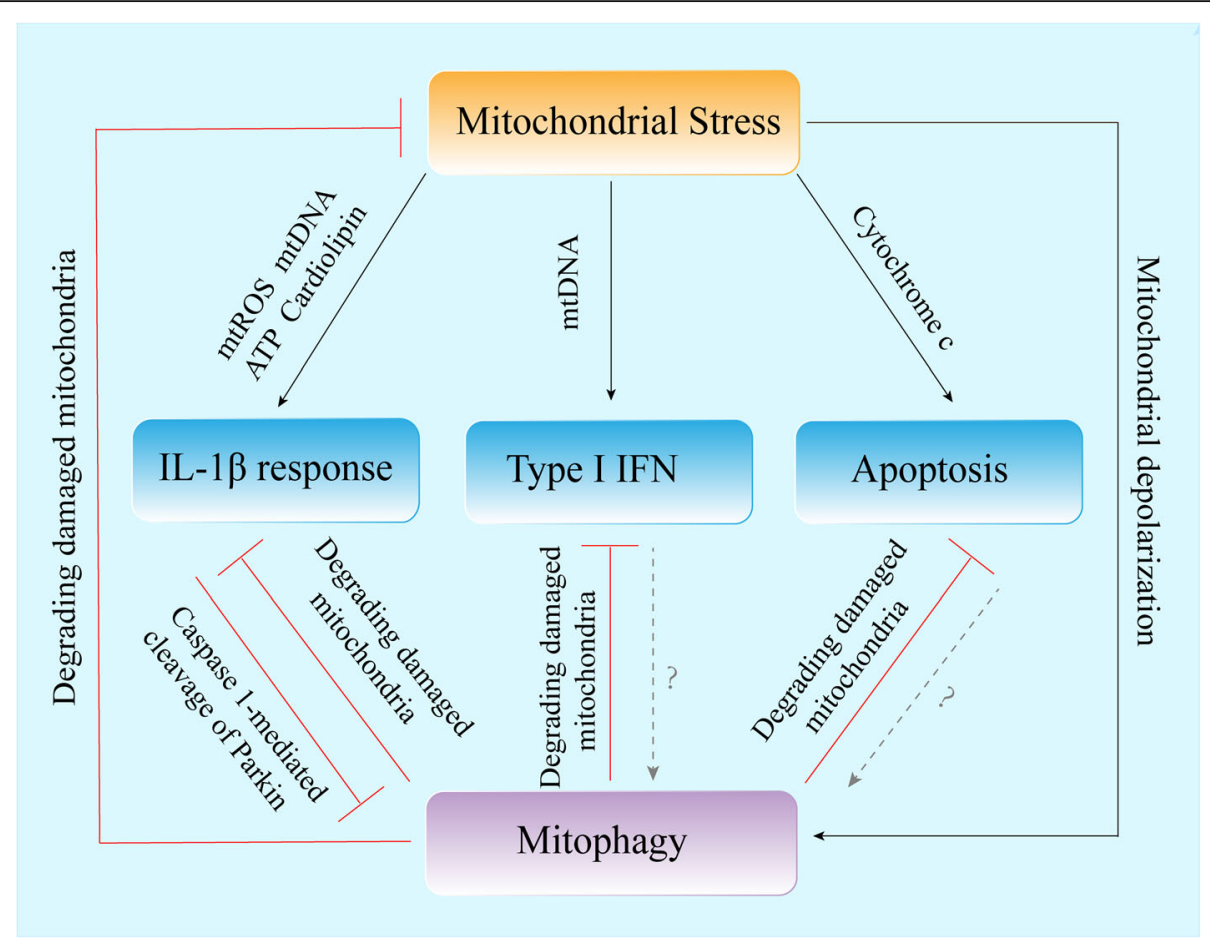

Fig. 3 Functions of mitophagy in innate immune responses triggered by mitochondrial stress. Mitochondrial stress triggers the release of mtDNA, mtROS, ATP and cytochrome $c$ into the cytosol and the externalization of cardiolipin, which lead to IL-1 $\beta$ secretion, type I IFN response and apoptosis. In addition, mitochondrial stress causes the dissipation of the mitochondrial membrane potential, which subsequently initiates the mitophagy process. Mitophagy attenuates the secretion of IL-1 $\beta$, type I IFN responses and apoptosis by degrading dysfunctional mitochondria. In turn, activated caspase-1 can inhibit mitophagy by cleaving Parkin

inhibit host cell apoptosis via eliminating damaged mitochondria to promote viral replication. Depletion of Drp1 and Parkin caused massive cytochrome $\mathrm{c}$ release into cytosol and promoted activation of caspase $-3 /-7$, induction of apoptosis [164].

Overall, mitophagy regulates immune responses triggered by mitochondrial stress, such as inflammasome activation, type I IFN responses and intrinsic apoptosis, through selective degrading dysfunctional mitochondria (Fig. 3). Furthermore, some pathogens can employ host mitophagy to avoid host immune defense. However, to date, our understanding of the role of mitophagy in the host innate immune and pathogenic immune evasion is limited, and further studies in vivo and in vitro are needed.

\section{Concluding remarks and future directions}

The present work reviews the pivotal function of mitophagy in the regulation of innate immune responses induced by mitochondrial stress. Critically, mitophagy can attenuate the activation of inflammasome, type I IFN responses and apoptosis triggered by mitochondrial stress via clearing dysfunctional mitochondria. Notably, viruses or bacteria can employ host mitophagy to avoid host immune responses. Despite many efforts in the past to understand the role of pathogens infection in the regulation of mitophagy, we are still far from a clear understanding as to what the mechanisms of pathogens hijack host mitophagy. A more thorough understanding of the underlying molecular mechanisms by which pathogens trigger and regulate mitophagy, especially crosstalk between virulence factors of pathogens and host mitochondrial-specific proteins will provide new ways to develop therapeutic strategies to fight infection and related diseases.

\section{Abbreviations}

PRRs: Pattern-recognition receptors; PAMPs: Pathogen-associated molecular patterns; TLRs: Toll-like receptors; NLRs: Nucleotide oligomerization domain (NOD)-like receptors; CLRs: C-type lectin receptors; RLRs: Retinoic acidinducible gene I (RIG-I)-like receptors; NF-kB: Nuclear factor-kappa B; MAPKs: Mitogen-activated protein kinases; IFN: Interferon; IRFs: Interferon regulatory factors; mtDNA: Mitochondrial DNA; OXPHOS: Oxidative phosphorylation system; ROS: Reactive oxygen species; DAMPs: Damageassociated molecular patterns; mtROS: Mitochondrial ROS; NLRC4: Nod-like receptor CARD domain containing 4; NLRP3: Nod-like receptor pyrin domain containing 3; AIM2: Absence in melanoma 2; LPS: Lipopolysaccharide;

TFAM: Mitochondrial transcription factor A; VDAC: Voltage-dependent anion channel; MPTP: Mitochondrial permeability transition pore;

MAVS: Mitochondrial antiviral signaling proteins; ISGs: Interferon-stimulated genes; cGAS: Cyclic guanosine monophosphate-adenosine monophosphate synthase; CGAMP: Cyclic guanosine monophosphate-adenosine monophosphate; STING: Stimulator of interferon genes; TBK1: TANK-binding kinase 1; Mtb: Mycobacterium tuberculosis; BMDM: Bone marrow-derived macrophages; Bax: $\mathrm{BCl}-2$ associated-X protein; Bak: BCL-2 homologous 
antagonist/killer; TLR9: Toll-like receptor 9; CpG: Cytosine-guanosine; pDCs: Plasmacytoid dendritic cells; NASH : Nonalcoholic steatohepatitis; SIRS: Sterile systemic inflammatory response syndrome; MDVs: Mitochondriaderived vesicles; OMM: Outer mitochondrial membrane; PPAR: Peroxisome proliferator-activated receptor; HBHA: Heparin-binding hemagglutinin; KLK12: Kallikrein 12; ER: Endoplasmic reticulum; Pink1: Phosphatase and tensin homologue (PTEN)-induced putative kinase 1; Parkin: Parkin RBR E3 ubiquitin protein ligase; IMM : Inner mitochondrial membrane; PARL: Presenilin-associated rhomboid-like; OPA1: Optic atrophy 1; MFN1/ 2: Mitofusin 1/2; OPTN : Optineurin; NDP52: Nuclear domain 10 protein 52; DRP1: Dynamin-related protein 1; AMFR/ Gp78: Autocrine motility factor receptor; SMURF1: SMAD-specific E3 ubiquitin-protein ligase 1;

MUL1: Mitochondrial E3 ubiquitin-protein ligase 1; SIAH1: Seven in absentia homolog 1; ARIH1: Ariadne RBR E3 Ub protein ligase 1; LIR : LC3-interacting region; BNIP3: BCL2 interacting protein 3; BNIP3L/NIX : BCL2 interacting protein 3 like; FUNDC1: FUN14 domain-containing protein 1; BCL2L13: BCL2 like 13; FKBP8: FKBP prolyl isomerase 8; RGC: Retinal ganglion cell; HIF1a: Hypoxia-inducible factor 1-alpha; PHB2: Prohibitin2; Atg16L1: Autophagy-related 16-like 1; HBV: Hepatitis B virus; HCV: Hepatitis C virus; MV-Edm: Measles virus of the Edmonston strain; HPIV3: Human parainfluenza virus type 3; TUFM: Tu translation elongation factor mitochondrial; HTNV: Hantavirus; L. monocytogenes: Listeria monocytogenes; RHDV: Rabbit haemorrhagic disease virus

\section{Acknowledgments}

Not applicable.

\section{Authors' contributions}

$Y S$ and $X Z$ conceived the manuscript, $Y S$ wrote the manuscript. $X Z$ and $Y Z$ critically reviewed the article before final submission. The authors read and approved the final manuscript.

\section{Funding}

This work was supported by National Natural Science Foundation of China (Project No. 31873005); China Agriculture Research System (Project No. CARS36); the National Key Research and Development Program (Project No. 2017YFD0500901)

\section{Availability of data and materials}

Not applicable.

\section{Ethics approval and consent to participate}

Not applicable.

\section{Consent for publication}

Not applicable.

\section{Competing interests}

The authors declare that they have no competing interests.

\section{Author details}

'Key Laboratory of Animal Epidemiology and Zoonosis, Ministry of Agriculture, National Animal Transmissible Spongiform Encephalopathy Laboratory, College of Veterinary Medicine, China Agricultural University, Beijing 100193, China. ${ }^{2}$ College of Animal Science, Southwest University, Chongqing 402460, China. ${ }^{3}$ Immunology Research Center, Medical Research Institute, Southwest University, Chongqing, China.

\section{Received: 6 July 2020 Accepted: 6 September 2020}

\section{Published online: 25 November 2020}

\section{References}

1. West AP, Shadel GS, Ghosh S. Mitochondria in innate immune responses. Nat Rev Immunol. 2011;11(6):389-402.

2. Fukata M, Vamadevan AS, Abreu MT. Toll-like receptors (TLRs) and nodlike receptors (NLRs) in inflammatory disorders. Semin Immunol. 2009; 21(4):242-53.

3. Marakalala MJ, Ndlovu H. Signaling C-type lectin receptors in antimycobacterial immunity. PLoS Pathog. 2017;13(6):e1006333.

4. Galluzzi L, Kepp O, Kroemer G. Mitochondria: master regulators of danger signalling. Nat Rev Mol Cell Biol. 2012;13(12):780-8.
5. Lane N, Martin W. The energetics of genome complexity. Nature. 2010; 467(7318):929-34.

6. Mclnerney J, Pisani D, O'Connell MJ. The ring of life hypothesis for eukaryote origins is supported by multiple kinds of data. Philos Trans R Soc Lond B Biol Sci. 2015;370(1678):20140323.

7. Shadel GS, Clayton DA. Mitochondrial DNA maintenance in vertebrates. Annu Rev Biochem. 1997;66:409-35.

8. Shadel GS, Horvath TL. Mitochondrial ROS signaling in organismal homeostasis. Cell. 2015;163(3):560-9.

9. Weinberg SE, Sena LA, Chandel NS. Mitochondria in the regulation of innate and adaptive immunity. Immunity. 2015;42(3):406-17.

10. West AP. Mitochondrial dysfunction as a trigger of innate immune responses and inflammation. Toxicology. 2017;391:54-63.

11. Lazarou M. Keeping the immune system in check: a role for mitophagy. Immunol Cell Biol. 2015:93(1):3-10.

12. Youle RJ, van der Bliek AM. Mitochondrial fission, fusion, and stress. Science. 2012;337(6098):1062-5.

13. De Duve C, Wattiaux R. Functions of lysosomes. Annu Rev Physiol. 1966;28: 435-92.

14. Youle RJ, Narendra DP. Mechanisms of mitophagy. Nat Rev Mol Cell Biol. 2011;12(1):9-14.

15. Sandhir R, Halder A, Sunkaria A. Mitochondria as a centrally positioned hub in the innate immune response. Biochim Biophys Acta. 2017;1863(5):1090-7.

16. Xu Y, Shen J, Ran Z. Emerging views of mitophagy in immunity and autoimmune diseases. Autophagy. 2019;16(1):3-17.

17. Wiens KE, Ernst JD. The mechanism for type I interferon induction by mycobacterium tuberculosis is bacterial strain-dependent. PLoS Pathog. 2016;12(8):e1005809.

18. Andreux PA, Houtkooper RH, Auwerx J. Pharmacological approaches to restore mitochondrial function. Nat Rev Drug Discov. 2013;12(6):465-83.

19. Barbour JA, Turner N. Mitochondrial stress signaling promotes cellular adaptations. Int J Cell Biol. 2014;2014:156020.

20. Baker MJ, Tatsuta T, Langer T. Quality control of mitochondrial proteostasis. Cold Spring Harb Perspect Biol. 2011;3(7):a007559.

21. Kim HE, Grant AR, Simic MS, Kohnz RA, Nomura DK, Durieux J, et al. Lipid biosynthesis coordinates a mitochondrial-to-cytosolic stress response. Cell. 2016;166(6):1539-52 e 16

22. Jovaisaite $\mathrm{V}$, Auwerx J. The mitochondrial unfolded protein responsesynchronizing genomes. Curr Opin Cell Biol. 2015;33:74-81.

23. Suen DF, Norris KL, Youle RJ. Mitochondrial dynamics and apoptosis. Genes Dev. 2008;22(12):1577-90.

24. Cheng $Y$, Ren $X$, Gowda AS, Shan Y, Zhang L, Yuan YS, et al. Interaction of Sirt3 with OGG1 contributes to repair of mitochondrial DNA and protects from apoptotic cell death under oxidative stress. Cell Death Dis. 2013;4: e731.

25. Singh B, Chatterjee A, Ronghe AM, Bhat NK, Bhat HK. Antioxidant-mediated up-regulation of OGG1 via NRF2 induction is associated with inhibition of oxidative DNA damage in estrogen-induced breast cancer. BMC Cancer. 2013;13:253.

26. Ni HM, Williams JA, Ding WX. Mitochondrial dynamics and mitochondrial quality control. Redox Biol. 2015:4:6-13.

27. Wai T, Langer T. Mitochondrial dynamics and metabolic regulation. Trends Endocrinol Metab. 2016;27(2):105-17.

28. Bock FJ, Tait SWG. Mitochondria as multifaceted regulators of cell death. Nat Rev Mol Cell Biol. 2020;21(2):85-100.

29. Gross O, Thomas CJ, Guarda G, Tschopp J. The inflammasome: an integrated view. Immunol Rev. 2011;243(1):136-51.

30. Latz E, Xiao TS, Stutz A. Activation and regulation of the inflammasomes. Nat Rev Immunol. 2013;13(6):397-411.

31. Martinon F, Mayor A, Tschopp J. The inflammasomes: guardians of the body. Annu Rev Immunol. 2009;27:229-65.

32. Schroder K, Tschopp J. The inflammasomes. Cell. 2010;140(6):821-32.

33. Wen H, Miao EA, Ting JP. Mechanisms of NOD-like receptor-associated inflammasome activation. Immunity. 2013;39(3):432-41.

34. Cruz CM, Rinna A, Forman HJ, Ventura AL, Persechini PM, Ojcius DM. ATP activates a reactive oxygen species-dependent oxidative stress response and secretion of proinflammatory cytokines in macrophages. J Biol Chem. 2007;282(5):2871-9.

35. Dostert C, Petrilli V, Van Bruggen R, Steele C, Mossman BT, Tschopp J. Innate immune activation through Nalp3 inflammasome sensing of asbestos and silica. Science. 2008;320(5876):674-7. 
36. Elliott El, Sutterwala FS. Initiation and perpetuation of NLRP3 inflammasome activation and assembly. Immunol Rev. 2015;265(1):35-52

37. Hornung V, Bauernfeind F, Halle A, Samstad EO, Kono H, Rock KL, et al. Silica crystals and aluminum salts activate the NALP3 inflammasome through phagosomal destabilization. Nat Immunol. 2008;9(8):847-56.

38. Martinon F, Petrilli V, Mayor A, Tardivel A, Tschopp J. Gout-associated uric acid crystals activate the NALP3 inflammasome. Nature. 2006; 440(7081):237-41.

39. Zhou R, Yazdi AS, Menu P, Tschopp J. A role for mitochondria in NLRP3 inflammasome activation. Nature. 2011;469(7329):221-5.

40. Nakahira K, Haspel JA, Rathinam VA, Lee SJ, Dolinay T, Lam HC, et al. Autophagy proteins regulate innate immune responses by inhibiting the release of mitochondrial DNA mediated by the NALP3 inflammasome. Nat Immunol. 2011;12(3):222-30.

41. Shimada K, Crother TR, Karlin J, Dagvadorj J, Chiba N, Chen S, et al. Oxidized mitochondrial DNA activates the NLRP3 inflammasome during apoptosis. Immunity. 2012;36(3):401-14.

42. Zhong Z, Liang S, Sanchez-Lopez E, He F, Shalapour S, Lin XJ, et al. New mitochondrial DNA synthesis enables NLRP3 inflammasome activation. Nature. 2018;560(7717):198-203.

43. Jabir MS, Hopkins L, Ritchie ND, Ullah I, Bayes HK, Li D, et al. Mitochondrial damage contributes to Pseudomonas aeruginosa activation of the inflammasome and is downregulated by autophagy. Autophagy. 2015;11(1): 166-82.

44. Bae JH, Jo SI, Kim SJ, Lee JM, Jeong JH, Kang JS, et al. Circulating cell-free mtDNA contributes to AIM2 Inflammasome-mediated chronic inflammation in patients with type 2 diabetes. Cells. 2019;8(4):328.

45. Tschopp J, Schroder K. NLRP3 inflammasome activation: the convergence of multiple signalling pathways on ROS production? Nat Rev Immunol. 2010; 10(3):210-5.

46. Iyer SS, Pulskens WP, Sadler JJ, Butter LM, Teske GJ, Ulland TK, et al. Necrotic cells trigger a sterile inflammatory response through the Nlrp3 inflammasome. Proc Natl Acad Sci U S A. 2009;106(48):20388-93.

47. Iyer SS, He Q, Janczy JR, Elliott El, Zhong Z, Olivier AK, et al. Mitochondrial cardiolipin is required for Nlrp3 inflammasome activation. Immunity. 2013; 39(2):311-23.

48. Toksoy A, Sennefelder H, Adam C, Hofmann S, Trautmann A, Goebeler M, et al. Potent NLRP3 Inflammasome activation by the HIV reverse transcriptase inhibitor Abacavir. J Biol Chem. 2017;292(7):2805-14.

49. Rasola A, Sciacovelli M, Pantic B, Bernardi P. Signal transduction to the permeability transition pore. FEBS Lett. 2010;584(10):1989-96.

50. Tomasello F, Messina A, Lartigue L, Schembri L, Medina C, Reina S, et al. Outer membrane VDAC1 controls permeability transition of the inner mitochondrial membrane in cellulo during stress-induced apoptosis. Cell Res. 2009;19(12):1363-76.

51. Subramanian N, Natarajan K, Clatworthy MR, Wang Z, Germain RN. The adaptor MAVS promotes NLRP3 mitochondrial localization and inflammasome activation. Cell. 2013;153(2):348-61.

52. West AP, Shadel GS. Mitochondrial DNA in innate immune responses and inflammatory pathology. Nat Rev Immunol. 2017;17(6):363-75.

53. Roers A, Hiller B, Hornung V. Recognition of endogenous nucleic acids by the innate immune system. Immunity. 2016:44(4):739-54.

54. Sun L, Wu J, Du F, Chen X, Chen ZJ. Cyclic GMP-AMP synthase is a cytosolic DNA sensor that activates the type I interferon pathway. Science. 2013; 339(6121):786-91.

55. Wu J, Sun L, Chen X, Du F, Shi H, Chen C, et al. Cyclic GMP-AMP is an endogenous second messenger in innate immune signaling by cytosolic DNA. Science. 2013;339(6121):826-30.

56. Zhao B, Du F, Xu P, Shu C, Sankaran B, Bell SL, et al. A conserved PLPLRT/SD motif of STING mediates the recruitment and activation of TBK1. Nature. 2019;569(7758):718-22.

57. Cai X, Chiu YH, Chen ZJ. The cGAS-cGAMP-STING pathway of cytosolic DNA sensing and signaling. Mol Cell. 2014;54(2):289-96.

58. Chen Q, Sun L, Chen ZJ. Regulation and function of the CGAS-STING pathway of cytosolic DNA sensing. Nat Immunol. 2016;17(10):1142-9.

59. Collins AC, Cai H, Li T, Franco LH, Li XD, Nair VR, et al. Cyclic GMP-AMP synthase is an innate immune DNA sensor for mycobacterium tuberculosis. Cell Host Microbe. 2015;17(6):820-8.

60. Gao D, Wu J, Wu YT, Du F, Aroh C, Yan N, et al. Cyclic GMP-AMP synthase is an innate immune sensor of HIV and other retroviruses. Science. 2013; 341(6148):903-6.
61. Watson RO, Bell SL, MacDuff DA, Kimmey JM, Diner EJ, Olivas J, et al. The cytosolic sensor cGAS detects mycobacterium tuberculosis DNA to induce type I Interferons and activate autophagy. Cell Host Microbe. 2015;17(6): $811-9$.

62. West AP, Khoury-Hanold W, Staron M, Tal MC, Pineda CM, Lang SM, et al. Mitochondrial DNA stress primes the antiviral innate immune response. Nature. 2015;520(7548):553-7.

63. Rongvaux A, Jackson R, Harman CC, Li T, West AP, de Zoete MR, et al. Apoptotic caspases prevent the induction of type I interferons by mitochondrial DNA. Cell. 2014;159(7):1563-77.

64. White MJ, McArthur K, Metcalf D, Lane RM, Cambier JC, Herold MJ, et al. Apoptotic caspases suppress mtDNA-induced STING-mediated type I IFN production. Cell. 2014;159(7):1549-62.

65. Ning $X$, Wang $Y$, Jing M, Sha M, Lv M, Gao P, et al. Apoptotic Caspases suppress type I interferon production via the cleavage of CGAS, MAVS, and IRF3. Mol Cell. 2019;74(1):19-31 e7.

66. Wang Y, Ning X, Gao P, Wu S, Sha M, Lv M, et al. Inflammasome activation triggers Caspase-1-mediated cleavage of CGAS to regulate responses to DNA virus infection. Immunity. 2017;46(3):393-404.

67. Barbalat R, Ewald SE, Mouchess ML, Barton GM. Nucleic acid recognition by the innate immune system. Annu Rev Immunol. 2011;29:185-214.

68. Garcia-Martinez I, Santoro N, Chen Y, Hoque R, Ouyang X, Caprio S, et al. Hepatocyte mitochondrial DNA drives nonalcoholic steatohepatitis by activation of TLR9. J Clin Invest. 2016;126(3):859-64.

69. Zhang JZ, Liu Z, Liu J, Ren JX, Sun TS. Mitochondrial DNA induces inflammation and increases TLR9/NF-kappaB expression in lung tissue. Int J Mol Med. 2014;33(4):817-24.

70. Caielli S, Athale S, Domic B, Murat E, Chandra M, Banchereau R, et al. Oxidized mitochondrial nucleoids released by neutrophils drive type I interferon production in human lupus. J Exp Med. 2016;213(5):697-713.

71. Carroll EC, Jin L, Mori A, Munoz-Wolf N, Oleszycka E, Moran HBT, et al. The vaccine adjuvant chitosan promotes cellular immunity via DNA sensor CGAS-STING-dependent induction of type I Interferons. Immunity. 2016; 44(3):597-608.

72. MCArthur K, Whitehead LW, Heddleston JM, Li L, Padman BS, Oorschot V, et al. BAK/BAX macropores facilitate mitochondrial herniation and mtDNA efflux during apoptosis. Science. 2018;359(6378):eaao6047.

73. Tait SW, Green DR. Mitochondria and cell death: outer membrane permeabilization and beyond. Nat Rev Mol Cell Biol. 2010;11(9):621-32.

74. Vandenabeele P, Galluzzi L, Vanden Berghe T, Kroemer G. Molecular mechanisms of necroptosis: an ordered cellular explosion. Nat Rev Mol Cell Biol. 2010;11(10):700-14.

75. Kale J, Osterlund EJ, Andrews DW. BCL-2 family proteins: changing partners in the dance towards death. Cell Death Differ. 2018;25(1):65-80.

76. Arnett E, Weaver AM, Woodyard KC, Montoya MJ, Li M, Hoang KV, et al. PPARgamma is critical for mycobacterium tuberculosis induction of $\mathrm{MCl}-1$ and limitation of human macrophage apoptosis. PLoS Pathog. 2018;14(6): e1007100

77. Tiku V, Tan MW, Dikic I. Mitochondrial functions in infection and immunity. Trends Cell Biol. 2020;30(4):263-75.

78. Sohn H, Kim JS, Shin SJ, Kim K, Won CJ, Kim WS, et al. Targeting of mycobacterium tuberculosis heparin-binding hemagglutinin to mitochondria in macrophages. PLoS Pathog. 2011;7(12):e1002435.

79. Lee Kl, Whang J, Choi HG, Son YJ, Jeon HS, Back YW, et al. Mycobacterium avium MAV2054 protein induces macrophage apoptosis by targeting mitochondria and reduces intracellular bacterial growth. Sci Rep. 2016;6: 37804.

80. Sabir N, Hussain T, Liao Y, Wang J, Song Y, Shahid M, et al. Kallikrein 12 regulates innate resistance of murine macrophages against Mycobacterium bovis infection by modulating autophagy and apoptosis. Cells. 2019;8(5):415.

81. Datta $D$, Khatri $P$, Singh A, Saha DR, Verma G, Raman $R$, et al. Mycobacterium fortuitum-induced ER-mitochondrial calcium dynamics promotes calpain/caspase-12/caspase-9 mediated apoptosis in fish macrophages. Cell Death Dis. 2018;4:30.

82. Chen M, Gan H, Remold HG. A mechanism of virulence: virulent mycobacterium tuberculosis strain H37Rv, but not attenuated H37Ra, causes significant mitochondrial inner membrane disruption in macrophages leading to necrosis. J Immunol. 2006;176(6):3707-16.

83. Danelishvili L, Everman J, Bermudez LE. Mycobacterium tuberculosis PPE68 and Rv2626c genes contribute to the host cell necrosis and bacterial escape from macrophages. Virulence. 2016;7(1):23-32. 
84. Guo W, Sun Y, Liu W, Wu X, Guo L, Cai P, et al. Small molecule-driven mitophagy-mediated NLRP3 inflammasome inhibition is responsible for the prevention of colitis-associated cancer. Autophagy. 2014;10(6):972-85.

85. Kim MJ, Bae SH, Ryu JC, Kwon Y, Oh JH, Kwon J, et al. SESN2/sestrin2 suppresses sepsis by inducing mitophagy and inhibiting NLRP3 activation in macrophages. Autophagy. 2016;12(8):1272-91.

86. Lupfer C, Thomas PG, Anand PK, Vogel P, Milasta S, Martinez J, et al. Receptor interacting protein kinase 2-mediated mitophagy regulates inflammasome activation during virus infection. Nat Immunol. 2013;14(5): 480-8.

87. Zhong Z, Umemura A, Sanchez-Lopez E, Liang S, Shalapour S, Wong J, et al. NF-kappaB restricts Inflammasome activation via elimination of damaged mitochondria. Cell. 2016;164(5):896-910.

88. Shibutani ST, Saitoh T, Nowag H, Munz C, Yoshimori T. Autophagy and autophagy-related proteins in the immune system. Nat Immunol. 2015; 16(10):1014-24.

89. Levine B, Kroemer G. Autophagy in the pathogenesis of disease. Cell. 2008; 132(1):27-42.

90. Levine B, Kroemer G. Biological functions of autophagy genes: a disease perspective. Cell. 2019;176(1-2):11-42.

91. Stolz A, Ernst A, Dikic I. Cargo recognition and trafficking in selective autophagy. Nat Cell Biol. 2014;16(6):495-501.

92. Palikaras K, Daskalaki I, Markaki M, Tavernarakis N. Mitophagy and agerelated pathologies: development of new therapeutics by targeting mitochondrial turnover. Pharmacol Ther. 2017;178:157-74.

93. Kondapalli C, Kazlauskaite A, Zhang N, Woodroof HI, Campbell DG, Gourlay $R$, et al. PINK1 is activated by mitochondrial membrane potential depolarization and stimulates Parkin E3 ligase activity by phosphorylating serine 65. Open Biol. 2012;2(5):120080.

94. Sekine S, Youle RJ. PINK1 import regulation; a fine system to convey mitochondrial stress to the cytosol. BMC Biol. 2018;16(1):2.

95. Koyano F, Okatsu K, Kosako H, Tamura Y, Go E, Kimura M, et al. Ubiquitin is phosphorylated by PINK1 to activate parkin. Nature. 2014;510(7503):162-6.

96. Gkikas I, Palikaras K, Tavernarakis N. The role of Mitophagy in innate immunity. Front Immunol. 2018;9:1283.

97. George S, Wang SM, Bi Y, Treidlinger M, Barber KR, Shaw GS, et al. Ubiquitin phosphorylated at Ser57 hyper-activates parkin. Biochim Biophys Acta Gen Subj. 2017:1861(11):3038-46.

98. Okatsu K, Koyano F, Kimura M, Kosako H, Saeki Y, Tanaka K, et al. Phosphorylated ubiquitin chain is the genuine Parkin receptor. J Cell Biol. 2015;209(1):111-28.

99. Ordureau A, Heo JM, Duda DM, Paulo JA, Olszewski JL, Yanishevski D, et al. Defining roles of PARKIN and ubiquitin phosphorylation by PINK1 in mitochondrial quality control using a ubiquitin replacement strategy. Proc Natl Acad Sci U S A. 2015;112(21):6637-42

100. Harper JW, Ordureau A, Heo JM. Building and decoding ubiquitin chains for mitophagy. Nat Rev Mol Cell Biol. 2018;19(2):93-108.

101. Matsuda N. Phospho-ubiquitin: upending the PINK-Parkin-ubiquitin cascade. J Biochem. 2016:159(4):379-85.

102. Bingol B, Tea JS, Phu L, Reichelt M, Bakalarski CE, Song Q, et al. The mitochondrial deubiquitinase USP30 opposes parkin-mediated mitophagy. Nature. 2014;510(7505):370-5.

103. Cornelissen T, Haddad D, Wauters F, Van Humbeeck C, Mandemakers W, Koentjoro B, et al. The deubiquitinase USP15 antagonizes Parkin-mediated mitochondrial ubiquitination and mitophagy. Hum Mol Genet. 2014;23(19): 5227-42.

104. Cunningham CN, Baughman JM, Phu L, Tea JS, Yu C, Coons M, et al. USP30 and parkin homeostatically regulate atypical ubiquitin chains on mitochondria. Nat Cell Biol. 2015;17(2):160-9.

105. Durcan TM, Tang MY, Perusse JR, Dashti EA, Aguileta MA, McLelland GL, et al. USP8 regulates mitophagy by removing K6-linked ubiquitin conjugates from parkin. EMBO J. 2014;33(21):2473-91.

106. Gersch M, Gladkova C, Schubert AF, Michel MA, Maslen S, Komander D. Mechanism and regulation of the Lys6-selective deubiquitinase USP30. Nat Struct Mol Biol. 2017;24(11):920-30.

107. Wang $Y$, Serricchio $M$, Jauregui $M$, Shanbhag $R$, Stoltz $T$, Di Paolo $C T$, et al. Deubiquitinating enzymes regulate PARK2-mediated mitophagy. Autophagy. 2015;11(4):595-606.

108. Wang L, Cho YL, Tang Y, Wang J, Park JE, Wu Y, et al. PTEN-L is a novel protein phosphatase for ubiquitin dephosphorylation to inhibit PINK1Parkin-mediated mitophagy. Cell Res. 2018;28(8):787-802.
109. Tilokani L, Nagashima S, Paupe V, Prudent J. Mitochondrial dynamics: overview of molecular mechanisms. Essays Biochem. 2018;62(3):341-60.

110. Pryde KR, Smith HL, Chau KY, Schapira AH. PINK1 disables the anti-fission machinery to segregate damaged mitochondria for mitophagy. J Cell Biol. 2016;213(2):163-71.

111. Tanaka A, Cleland MM, Xu S, Narendra DP, Suen DF, Karbowski M, et al. Proteasome and p97 mediate mitophagy and degradation of mitofusins induced by Parkin. J Cell Biol. 2010;191(7):1367-80.

112. Chen Y, Dorn GW 2nd. PINK1-phosphorylated mitofusin 2 is a Parkin receptor for culling damaged mitochondria. Science. 2013;340(6131):471-5.

113. McLelland GL, Fon EA. MFN2 retrotranslocation boosts mitophagy by uncoupling mitochondria from the ER. Autophagy. 2018;14(9):1658-60.

114. McLelland GL, Goiran T, Yi W, Dorval G, Chen CX, Lauinger ND, et al. Mfn2 ubiquitination by PINK1/parkin gates the p97-dependent release of ER from mitochondria to drive mitophagy. Elife. 2018;7:e32866.

115. Di Rita A, Peschiaroli A, Acunzo PD, Strobbe D, Hu Z, Gruber J, et al. HUWE1 E3 ligase promotes PINK1/PARKIN-independent mitophagy by regulating AMBRA1 activation via IKKalpha. Nat Commun. 2018;9(1):3755.

116. Fu M, St-Pierre P, Shankar J, Wang PT, Joshi B, Nabi IR. Regulation of mitophagy by the Gp78 E3 ubiquitin ligase. Mol Biol Cell. 2013;24(8):1153-62.

117. Orvedahl A, Sumpter R Jr, Xiao G, Ng A, Zou Z, Tang Y, et al. Image-based genome-wide siRNA screen identifies selective autophagy factors. Nature. 2011:480(7375):113-7.

118. Li J, Qi W, Chen G, Feng D, Liu J, Ma B, et al. Mitochondrial outermembrane E3 ligase MUL1 ubiquitinates ULK1 and regulates seleniteinduced mitophagy. Autophagy. 2015;11(8):1216-29.

119. Szargel R, Shani V, Abd Elghani F, Mekies LN, Liani E, Rott R, et al. The PINK1, synphilin-1 and SIAH-1 complex constitutes a novel mitophagy pathway. Hum Mol Genet. 2016;25(16):3476-90.

120. Villa E, Proics E, Rubio-Patino C, Obba S, Zunino B, Bossowski JP, et al. Parkin-independent Mitophagy controls chemotherapeutic response in cancer cells. Cell Rep. 2017;20(12):2846-59.

121. Chen Z, Liu L, Cheng Q, Li Y, Wu H, Zhang W, et al. Mitochondrial E3 ligase MARCH5 regulates FUNDC1 to fine-tune hypoxic mitophagy. EMBO Rep. 2017;18(3):495-509.

122. Zhang H, Bosch-Marce M, Shimoda LA, Tan YS, Baek JH, Wesley JB, et al. Mitochondrial autophagy is an HIF-1-dependent adaptive metabolic response to hypoxia. J Biol Chem. 2008;283(16):10892-903.

123. Sandoval H, Thiagarajan P, Dasgupta SK, Schumacher A, Prchal JT, Chen M, et al. Essential role for nix in autophagic maturation of erythroid cells. Nature. 2008;454(7201):232-5.

124. Liu L, Feng D, Chen G, Chen M, Zheng Q, Song P, et al. Mitochondrial outer-membrane protein FUNDC1 mediates hypoxia-induced mitophagy in mammalian cells. Nat Cell Biol. 2012;14(2):177-85.

125. Murakawa T, Yamaguchi O, Hashimoto A, Hikoso S, Takeda T, Oka T, et al. Bcl-2-like protein 13 is a mammalian Atg32 homologue that mediates mitophagy and mitochondrial fragmentation. Nat Commun. 2015;6:7527.

126. Bhujabal Z, Birgisdottir AB, Sjottem E, Brenne HB, Overvatn A, Habisov S, et al. FKBP8 recruits $L C 3 A$ to mediate Parkin-independent mitophagy. EMBO Rep. 2017;18(6):947-61

127. Zhang Y, Yao Y, Qiu X, Wang G, Hu Z, Chen S, et al. Listeria hijacks host mitophagy through a novel mitophagy receptor to evade killing. Nat Immunol. 2019:20(4):433-46.

128. Moore CB, Bergstralh DT, Duncan JA, Lei Y, Morrison TE, Zimmermann AG, et al. NLRX1 is a regulator of mitochondrial antiviral immunity. Nature. 2008; 451(7178):573-7.

129. Tattoli I, Carneiro LA, Jehanno M, Magalhaes JG, Shu Y, Philpott DJ, et al. NLRX1 is a mitochondrial NOD-like receptor that amplifies NF-kappaB and JNK pathways by inducing reactive oxygen species production. EMBO Rep. 2008:9(3):293-300.

130. Zhang J, Ney PA. Role of BNIP3 and NIX in cell death, autophagy, and mitophagy. Cell Death Differ. 2009;16(7):939-46.

131. O'Sullivan TE, Johnson LR, Kang HH, Sun JC. BNIP3- and BNIP3L-mediated Mitophagy promotes the generation of natural killer cell memory. Immunity. 2015;43(2):331-42

132. Hanna RA, Quinsay MN, Orogo AM, Giang K, Rikka S, Gustafsson AB. Microtubule-associated protein 1 light chain 3 (LC3) interacts with Bnip3 protein to selectively remove endoplasmic reticulum and mitochondria via autophagy. J Biol Chem. 2012;287(23):19094-104.

133. Zhu Y, Massen S, Terenzio M, Lang V, Chen-Lindner S, Eils R, et al. Modulation of Serines 17 and 24 in the LC3-interacting region of Bnip3 
determines pro-survival MitophagyversusApoptosis. J Biol Chem. 2013; 288(2):1099-113.

134. Esteban-Martinez L, Boya P. BNIP3L/NIX-dependent mitophagy regulates cell differentiation via metabolic reprogramming. Autophagy. 2018;14(5):915-7.

135. Yuan $Y$, Zheng $Y$, Zhang X, Chen Y, Wu X, Wu J, et al. BNIP3L/NIX-mediated mitophagy protects against ischemic brain injury independent of PARK2. Autophagy. 2017;13(10):1754-66.

136. Gupta SS, Sharp R, Hofferek C, Kuai L, Dorn GW 2nd, Wang J, et al. NIXmediated Mitophagy promotes effector memory formation in antigenspecific CD8(+) T cells. Cell Rep. 2019;29(7):1862-77 e7.

137. Lampert MA, Orogo AM, Najor RH, Hammerling BC, Leon $L$, Wang BJ, et al. BNIP3L/NIX and FUNDC1-mediated mitophagy is required for mitochondrial network remodeling during cardiac progenitor cell differentiation. Autophagy. 2019;15(7):1182-98.

138. Wu W, Tian W, Hu Z, Chen G, Huang L, Li W, et al. ULK1 translocates to mitochondria and phosphorylates FUNDC1 to regulate mitophagy. EMBO Rep. 2014;15(5):566-75

139. Chen M, Chen Z, Wang Y, Tan Z, Zhu C, Li Y, et al. Mitophagy receptor FUNDC1 regulates mitochondrial dynamics and mitophagy. Autophagy. 2016;12(4):689-702.

140. Wu W, Li W, Chen $H$, Jiang $L$, Zhu R, Feng D. FUNDC1 is a novel mitochondrial-associated-membrane (MAM) protein required for hypoxiainduced mitochondrial fission and mitophagy. Autophagy. 2016;12(9):16756.

141. Yoo SM, Yamashita SI, Kim H, Na D, Lee H, Kim SJ, et al. FKBP8 LIRLdependent mitochondrial fragmentation facilitates mitophagy under stress conditions. FASEB J. 2020;34(2):2944-57.

142. Chu CT, Ji J, Dagda RK, Jiang JF, Tyurina YY, Kapralov AA, et al. Cardiolipin externalization to the outer mitochondrial membrane acts as an elimination signal for mitophagy in neuronal cells. Nat Cell Biol. 2013;15(10):1197-205.

143. Wei Y, Chiang WC, Sumpter R Jr, Mishra P, Levine B. Prohibitin 2 is an inner mitochondrial membrane Mitophagy receptor. Cell. 2017;168(1-2):224-38 e10.

144. Merkwirth C, Langer T. Prohibitin function within mitochondria: essential roles for cell proliferation and cristae morphogenesis. Biochim Biophys Acta. 2009;1793(1):27-32.

145. Lahiri $\vee$, Klionsky DJ. PHB2/prohibitin 2: an inner membrane mitophagy receptor. Cell Res. 2017;27(3):311-2.

146. Zhang T, Xue L, Li L, Tang C, Wan Z, Wang R, et al. BNIP3 protein suppresses PINK1 kinase Proteolytic cleavage to promote Mitophagy. J Biol Chem. 2016;291(41):21616-29.

147. Yan C, Gong L, Chen L, Xu M, Abou-Hamdan H, Tang M, et al. PHB2 (prohibitin 2) promotes PINK1-PRKN/Parkin-dependent mitophagy by the PARL-PGAM5-PINK1 axis. Autophagy. 2019;16(3):419-34.

148. Kim M-J, Yoon J-H, Ryu J-H. Mitophagy: a balance regulator of NLRP3 inflammasome activation. BMB Rep. 2016;49(10):529-35.

149. Saitoh T, Fujita N, Jang MH, Uematsu S, Yang BG, Satoh T, et al. Loss of the autophagy protein Atg16L1 enhances endotoxin-induced IL-1beta production. Nature. 2008:456(7219):264-8.

150. Chen K, Feng L, Hu W, Chen J, Wang X, Wang L, et al. Optineurin inhibits NLRP3 inflammasome activation by enhancing mitophagy of renal tubular cells in diabetic nephropathy. FASEB J. 2019;33(3):4571-85.

151. Li W, Li Y, Siraj S, Jin H, Fan Y, Yang X, et al. FUN14 domain-containing 1mediated Mitophagy suppresses Hepatocarcinogenesis by inhibition of Inflammasome activation in mice. Hepatology. 2019;69(2):604-21.

152. Crespo I, Fernandez-Palanca P, San-Miguel B, Alvarez M, Gonzalez-Gallego J, Tunon MJ. Melatonin modulates mitophagy, innate immunity and circadian clocks in a model of viral-induced fulminant hepatic failure. J Cell Mol Med. 2020:24:7625-36.

153. Li J, Ma C, Long F, Yang D, Liu X, Hu Y, et al. Parkin impairs antiviral immunity by suppressing the mitochondrial reactive oxygen species-Nlrp3 Axis and antiviral inflammation. iScience. 2019;16:468-84

154. Yu J, Nagasu H, Murakami T, Hoang H, Broderick L, Hoffman HM, et al. Inflammasome activation leads to Caspase-1-dependent mitochondria damage and block of mitophagy. Proc Natl Acad Sci U S A. 2014;111(43): 15514-9.

155. Ivashkiv LB, Donlin LT. Regulation of type I interferon responses. Nat Rev Immunol. 2014;14(1):36-49.

156. Zhang $L$, Qin $Y$, Chen M. Viral strategies for triggering and manipulating mitophagy. Autophagy. 2018;14(10):1665-73.
157. Xia M, Gonzalez P, Li C, Meng G, Jiang A, Wang H, et al. Mitophagy enhances oncolytic measles virus replication by mitigating DDX58/RIG-I-like receptor signaling. J Virol. 2014;88(9):5152-64.

158. Khan M, Syed GH, Kim SJ, Siddiqui A. Hepatitis B virus-induced Parkindependent recruitment of linear ubiquitin assembly complex (LUBAC) to mitochondria and attenuation of innate immunity. PLoS Pathog. 2016;12(6): e1005693.

159. Ding B, Zhang L, Li Z, Zhong Y, Tang Q, Qin Y, et al. The matrix protein of human Parainfluenza virus type 3 induces Mitophagy that suppresses interferon responses. Cell Host Microbe. 2017;21(4):538-47 e4.

160. Wang K, Ma H, Liu H, Ye W, Li Z, Cheng L, et al. The glycoprotein and Nucleocapsid protein of hantaviruses manipulate autophagy flux to restrain host innate immune responses. Cell Rep. 2019;27(7):2075-91 e5.

161. Qi N, Shi Y, Zhang R, Zhu W, Yuan B, Li X, et al. Multiple truncated isoforms of MAVS prevent its spontaneous aggregation in antiviral innate immune signalling. Nat Commun. 2017:8:15676.

162. Sliter DA, Martinez J, Hao L, Chen X, Sun N, Fischer TD, et al. Parkin and PINK1 mitigate STING-induced inflammation. Nature. 2018;561(7722):258-62.

163. Kim SJ, Khan M, Quan J, Till A, Subramani S, Siddiqui A. Hepatitis B virus disrupts mitochondrial dynamics: induces fission and mitophagy to attenuate apoptosis. PLoS Pathog. 2013;9(12):e1003722.

164. Kim SJ, Syed GH, Khan M, Chiu WW, Sohail MA, Gish RG, et al. Hepatitis C virus triggers mitochondrial fission and attenuates apoptosis to promote viral persistence. Proc Natl Acad Sci U S A. 2014;111(17):6413-8.

\section{Publisher's Note}

Springer Nature remains neutral with regard to jurisdictional claims in published maps and institutional affiliations.
Ready to submit your research? Choose BMC and benefit from:

- fast, convenient online submission

- thorough peer review by experienced researchers in your field

- rapid publication on acceptance

- support for research data, including large and complex data types

- gold Open Access which fosters wider collaboration and increased citations

- maximum visibility for your research: over $100 \mathrm{M}$ website views per year

At BMC, research is always in progress.

Learn more biomedcentral.com/submissions 